\title{
Optimum design of tuned mass damper systems for seismic structures
}

\author{
I. Abdulsalam, M. Al-Janabi \& M. G. Al-Taweel \\ Department of Civil Engineering, Faculty of Engineering, \\ Altahadi University, Sirte, Libya
}

\begin{abstract}
Tuned mass dampers are well known devices for the passive control of vibrations in buildings subjected to earthquake loadings. Various methods have been proposed for the design of tuned mass damper (TMD) systems. In the present work, a method is suggested for obtaining the values of the parameters required for designing an efficient TMD system when attached to a SDOF system. In this method, the values of the optimum frequency ratio and optimum damping ratio for the TMD system are defined as the values that will reduce the maximum displacement of the structure to a minimum value when subjected to a specific earthquake time-acceleration history. For this purpose, a MATLAB computer program is developed. The program consists of a dynamic analysis subroutine embedded in a nonlinear constrained optimization program. The suggested method is used in selected case studies showing its efficiency when compared to other methods for designing TMD systems attached to SDOF systems.
\end{abstract}

Keywords: tuned mass damper, control of structures, optimum design, earthquake time-history, optimum design.

\section{Introduction}

The tuned mass damper (TMD) system represents an important type of passive control devices of structures subjected to dynamic loads. It can be installed to new or existing structures to improve their resistance to earthquakes and winds. A TMD system consists of a mass, a spring and a damper. If these properties are properly designed and selected, then the TMD device can be effective in suppressing undesirable vibrations induced by earthquake or wind loads. 
Obtaining the optimal design for a TMD system has been the goal of many researchers for many decades. In these researches different assumptions have been made regarding the simulation of the acting dynamic force, its location and the criteria used in defining the optimal design parameters. Den Hartog [1] has derived the formula for the optimum values of the TMD parameters for a SDOF structure when subjected to a harmonic load. An extension has been made by Warburton and Ayorinde [2] and Tsai and Lin [3], where damping in the main mass was considered and several types of harmonic excitations were examined. Extensive research was also made by Warburton [4] and Rana and Soong [5], where formulas for several types of excitations were developed. In this case the harmonic and random excitations were applied either on the main system or at the base of the structure. Sadek et al. [6] suggested a method for estimating the design parameters of TMDs for seismic applications, the criterion used to obtain the optimum parameters was to select, for a given mass ratio, the frequency and damping ratios that would result in equal and large modal damping in the first two modes of vibration.

As can be noticed from the brief literature review, various assumptions have been made regarding the earthquake loading (Harmonic or Random), and about the location of the acting force (on the structure or at its base). In the present work, a method is suggested to obtain the optimum TMD parameters. In this method, earthquake records are used to obtain the optimal TMD properties when subjected to the earthquake forces at its base.

\section{Description of the suggested method}

Consider the TMD system with mass $\boldsymbol{m}_{\boldsymbol{d}}$, spring stiffness $\boldsymbol{k}_{\boldsymbol{d}}$ and damping coefficient $\boldsymbol{c}_{\boldsymbol{d}}$ shown in Figure 1 attached to a SDOF structure with a stiffness $\boldsymbol{K}$, mass $\boldsymbol{M}$ and a structure damping coefficient $\boldsymbol{C}$. The resulting overall structure will have two degrees of freedom. The basic features of the method suggested for obtaining the optimum design parameters of a TMD system are as follows.

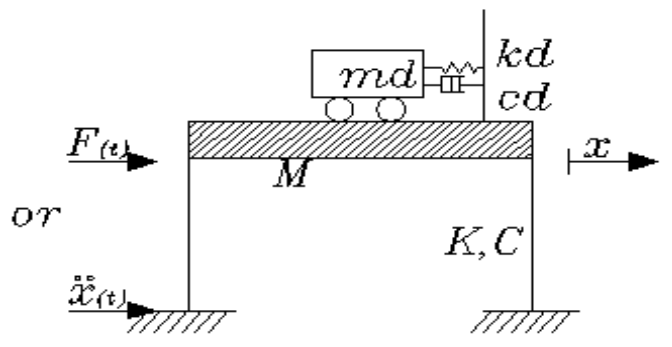

Figure 1: SDOF structures with the TMD system. 


\subsection{Excitation force}

In order to obtain the optimum design parameters of the TMD system certain assumptions regarding the excitation force should be made. To simulate actual behavior, it is assumed in this study that the structure is subjected to base excitation. The excitation force vector is computed from time acceleration history for a given earthquake. In the present work all time acceleration histories are taken from actual earthquake records. It should be noted that research is developing about generating ground earthquake time histories for design (Fengxin et al. [7], Abdalla and Hag-Elhassam [8] and Varpasuo and Gelder [9]).

\subsection{Optimization criterion and optimization parameters}

In the present work, the optimization criterion and parameters used by many authors is adopted (Den Hartog [1], Rana and Soong [5] and Tsai and Lin [3]). In this criterion, the optimum design parameters $\boldsymbol{k}_{\boldsymbol{d}}, \boldsymbol{c}_{\boldsymbol{d}}$ for a given $\boldsymbol{m}_{\boldsymbol{d}}$ are defined as those values that minimize the maximum relative displacement of the structure when subjected to an excitation. The maximum relative displacement of a regular SDOF shear building frame usually occurs at the top.

\subsection{Statement of the problem as a constrained nonlinear optimization problem}

Figure 2 shows a shear building structure provided with TMD system at the top floor. When the structure is subjected to a given earthquake excitation (acceleration-time $\ddot{\boldsymbol{x}}_{g}$ ), then $\boldsymbol{u}_{t}$ is the relative displacement occurring at the top of the frame.

Defining:

$\boldsymbol{u}_{t \operatorname{tmax}}$ : Maximum $\boldsymbol{u}_{\boldsymbol{t}}$ occurring during the earthquake duration.

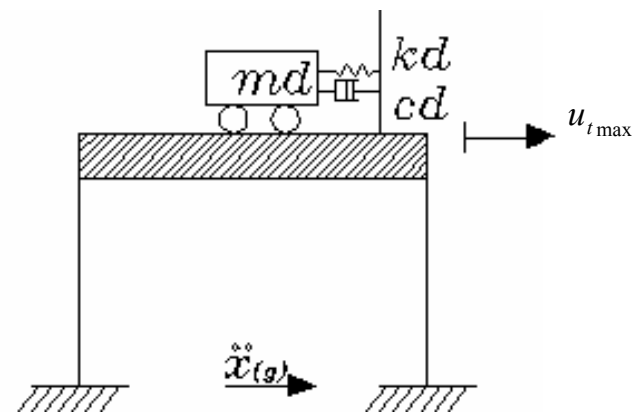

Figure 2: Shear building under earthquake excitation with the TMD system.

For a given total structure properties $([\boldsymbol{K}],[\boldsymbol{C}],[\boldsymbol{M}])$, TMD mass $\boldsymbol{m}_{\boldsymbol{d}}$ and earthquake excitation $\ddot{\boldsymbol{x}}_{g}, \boldsymbol{u}_{\boldsymbol{t} \max }$ will be function of $\boldsymbol{c}_{\boldsymbol{d}}, \boldsymbol{k}_{\boldsymbol{d}}$ only. This problem can 
be put in the following optimization problem: Find $\boldsymbol{c}_{\boldsymbol{d}}, \boldsymbol{k}_{\boldsymbol{d}}$ that minimizes the following objective function:

$$
\boldsymbol{u}_{\text {tmax }}=\mathrm{f}\left(\boldsymbol{c}_{\boldsymbol{d}}, \boldsymbol{k}_{\boldsymbol{d}}\right) \text { subjected to the inequality constrains } \boldsymbol{c}_{\boldsymbol{d}}>0 \text { and } \boldsymbol{k}_{\boldsymbol{d}}>0 \text {. }
$$

This problem can be classified as multivariable, nonlinear constrained minimization problem. For the treatment of such problem, one of the functions available in the MATLAB [10] optimization toolbox is used.

\subsection{Developed computer program for optimum design of a TMD system}

As a first stage, a MATLAB computer program is developed for the analysis of SDOF structure with TMD system when subjected to earthquake excitation. This program is based on Newmark's method. As a second stage, the above mentioned program is embedded in another nonlinear constrained optimization MATLAB program. This last program is used to obtain the optimum design parameters of the TMD system as mentioned in previous section. Details of the MATLAB software are given in [11].

\subsection{Convergence of the proposed method to the optimum solution}

To demonstrate the capability of the proposed method to catch the optimum design parameters for a TMD system when attached to structures, many problems are examined (Al-Taweel [11]). One of these verification problems is discussed herein. A single story shear building with properties shown in Figure 3 is considered. A TMD is attached to the top with mass $\boldsymbol{m}_{\boldsymbol{d}}=1.5$ ton equal to $3 \%$ of the total mass of the structure.

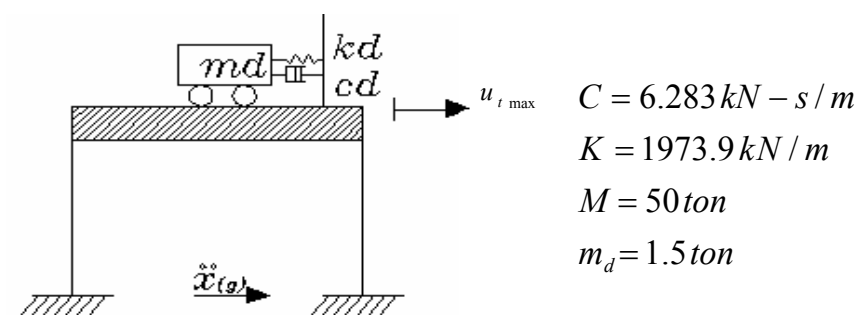

Figure 3: Structure studied as verification problem.

The objective is to determine the optimum value of TMD stiffness $\boldsymbol{k}_{\boldsymbol{d}}$ and damping $\boldsymbol{c}_{\boldsymbol{d}}$ that will minimize displacement $\boldsymbol{u}_{\text {tmax }}$ at the top when the structure is subjected to El-Centro earthquake excitation. To understand the variation of $\boldsymbol{u}_{\text {tmax }}$ with various values of $\boldsymbol{k}_{\boldsymbol{d}}$ and $\boldsymbol{c}_{\boldsymbol{d}}$ the first stage software is used to compute $\boldsymbol{u}_{\text {tmax }}$ for $\boldsymbol{k}_{\boldsymbol{d}}$ (40 to $70 \mathrm{kN} / \mathrm{m}$ with steps of .2) and $\boldsymbol{c}_{\boldsymbol{d}}$ (0 to $1.9 \mathrm{kN}-\mathrm{s} / \mathrm{m}$ with steps of $0.1)$. The results are plotted as three-dimensional function surface $\left[\boldsymbol{u}_{\text {tmax }}=\mathrm{f}\left(\boldsymbol{c}_{\boldsymbol{d}}\right.\right.$, $\left.\boldsymbol{k}_{\boldsymbol{d}}\right)$ ] in Figure 4 and as contour lines as shown in Figure 5. Next, the second stage software is used to obtain the optimum design parameters through 
minimization process, as described in previous sections. For the minimization process it is given that for this problem, the upper bound and the lower bound value of the stiffness $\boldsymbol{k}_{\boldsymbol{d}}$ are 0 and $1000 \mathrm{kN} / \mathrm{m}$ respectively, while the upper bound and lower bound of the damping are 0 and $100 \mathrm{kN}-\mathrm{s} / \mathrm{m}$ respectively. After running the problem, it is found that the optimum values of stiffness and damping of the TMD are $\boldsymbol{k}_{\boldsymbol{d}}=54.08 \mathrm{kN} / \mathrm{m}$ and $\boldsymbol{c}_{\boldsymbol{d}}=0.643 \mathrm{kN}-\mathrm{s} / \mathrm{m}$. The corresponding value of $\boldsymbol{u}_{\text {tmax }}$ is $0.1179 \mathrm{~m}$. When projecting these results on the contour plot in Figure 5 it can clearly noticed that the solution given by the second stage optimization software represents the minimum value for the surface or contour plot shown in Figures 5 and 6 . This proves the capability of the software to catch the minimum value of $\boldsymbol{u}_{\text {tmax }}$ and the corresponding optimum values of $\boldsymbol{k}_{\boldsymbol{d}}$ and $\boldsymbol{c}_{\boldsymbol{d}}$.

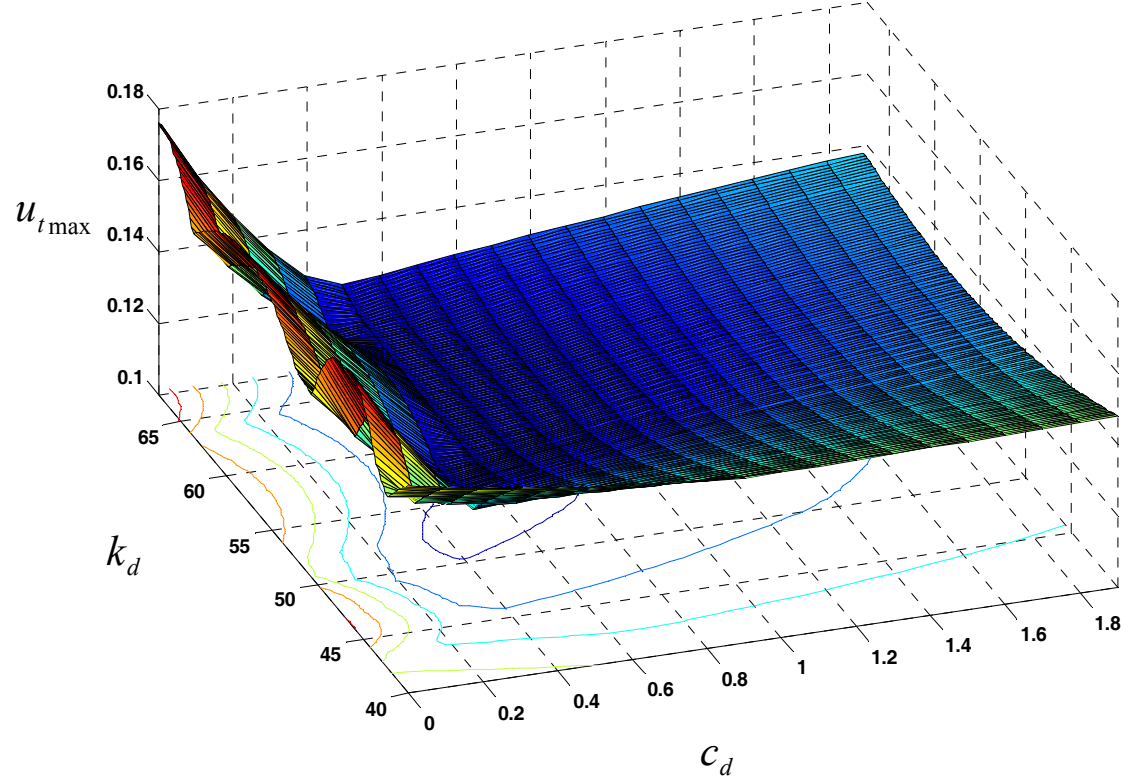

Figure 4: Variation of $u_{t_{\max }}$ with $k_{d}$ and $c_{d}$ for the example in Figure 3 as a three dimensional surface.

\section{Case study one: efficiency of the present study method in reducing SDOF structure vibrations under earthquake excitations}

In this case, a SDOF structures with $\mathbf{M}=30$ Ton, $\boldsymbol{K}=2700 \mathrm{kN} / \mathrm{m}(\mathrm{f}=1.5 \mathrm{~Hz})$ is studied. TMD systems with $3 \%$ mass ratio are tuned to control vibrations in the structure when subjected to 18 earthquake records. These earthquake records cover a wide range of earthquake dominant frequency (from 0.3 to $4.82 \mathrm{~Hz}$.). The properties of TMD systems $\boldsymbol{k}_{\boldsymbol{d}}, \boldsymbol{c}_{\boldsymbol{d}}$ are computed using three methods (as shown in Figure 6) and the present study method using the MATLAB program 
developed in this work. This was repeated for each of the 18 earthquakes excitations. Figure 6 shows the variation of percentage reduction in maximum displacement $\boldsymbol{u}_{\text {tmax }}$ with earthquake dominant frequency for the investigated structure. The results showed that for all 18 Earthquake records the

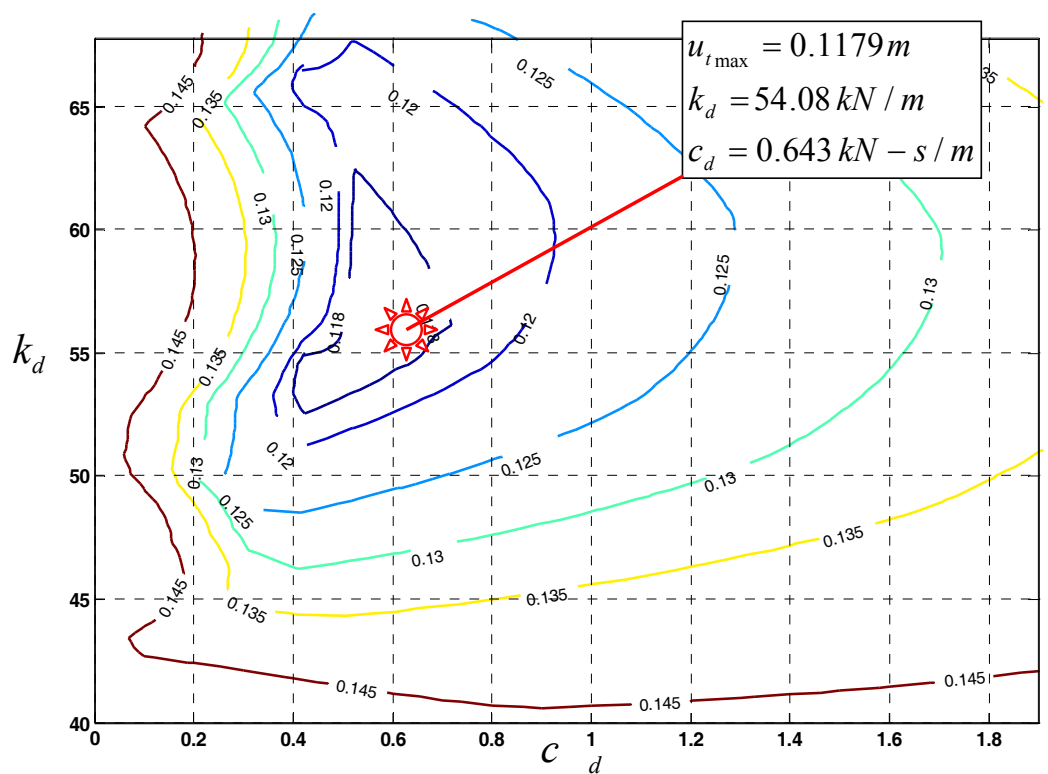

Figure 5: Variation of $u_{t_{\max }}$ with $k_{d}$ and $c_{d}$ for the example in Figure 3 as a contour line.

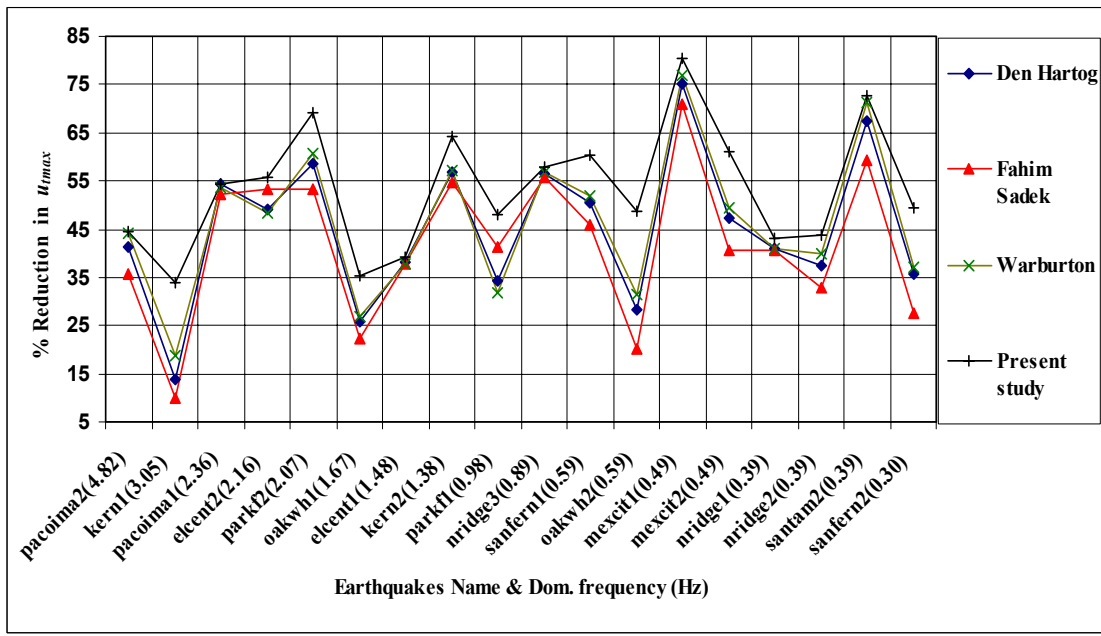

Figure 6: Percentage of reduction in $u_{t_{\max }}$ for various earthquake records for $\boldsymbol{f}=1.5 \mathrm{~Hz}$. 
method proposed in this study gave the maximum reduction in $\boldsymbol{u}_{\text {tmax }}$. On the contrary to other methods, the present study method gave different values for the optimums $\boldsymbol{k}_{\boldsymbol{d}}, \boldsymbol{c}_{\boldsymbol{d}}$ because it considers the earthquake characteristic in obtaining these optimum values. The figure also shows that the efficiency of the TMD system for all methods may vary with the characteristic of earthquakes. The maximum reduction in displacement observed is about $80 \%$ in this case study.

\section{Case study two: effect of TMD mass ratio on the present study results}

The main purpose here is to investigate the effect of the variation of TMD mass ratio on the optimum values of TMD frequency ratio $f_{\text {dopt }}$ and TMD damping ratio $\boldsymbol{\xi}_{\text {dopt }}$ when computed according to the present study method. Figure 7 shows the SDOF structures used in the present investigation. A TMD system with mass ratio ranging from 0 to 0.1 is attached to the structure. Also, three earthquake records are used in the study. The developed MATLAB software is used to compute the optimum $\boldsymbol{\xi}_{\text {dopt }}$ and $\boldsymbol{f}_{\text {dopt }}$.

$$
\begin{aligned}
& \omega=\sqrt{\frac{K}{M}} \quad \omega_{d}=\sqrt{\frac{k_{d}}{m_{d}}} \\
& \xi=\frac{C}{2 \omega M} \quad \xi_{d}=\frac{c_{d}}{2 \omega_{d} m_{d}} \\
& \mu=\frac{m_{d}}{M} \quad f_{d}=\frac{\omega_{d}}{\omega} \quad \text { गा7ग } \quad \overrightarrow{\ddot{x} g} \quad \text { गा7ा } \\
& \mu=(0-0.1) \\
& m_{d}=(0-4) \text { ton } \\
& \xi=0 \\
& K=(9870) \mathrm{kN} / \mathrm{m} \\
& f=2.5 \mathrm{~Hz}
\end{aligned}
$$

Figure 7: $\quad$ Structure studied in the case study.

Figure 8 shows the effect of TMD mass ratio variation on the optimum TMD frequency ratio $f_{\text {dopt }}$ for three earthquake excitations. The figures also show the variation of $\boldsymbol{f}_{\text {dopt }}$ with $\boldsymbol{\mu}$ as computed by the Den Hartog method. The results show that this relation is greatly affected by the type earthquake excitation to the extent that there is no unique shape for this relation as given by the Den Hartog Method. Similar behavior is also noticed for the relation between $\boldsymbol{\xi}_{\text {dopt }}$ and the mass ratio $\boldsymbol{\mu}$ as shown in Figure 9. The important conclusion from the above mentioned figures is that the relation of $\boldsymbol{f}_{\text {dopt }}$ or $\boldsymbol{\xi}_{\text {dopt }}$ with $\boldsymbol{\mu}$ is highly affected by the earthquake type and cannot predicted by simple equations as in the Den Hartog or other similar methods.

Figure 10 shows the variation of maximum displacement $\boldsymbol{u}_{\text {tmax }}$ of the structure (when controlled with $\boldsymbol{\xi}_{\text {dopt }}$ and $\boldsymbol{f}_{\text {dopt }}$ ) with mass ratio $\boldsymbol{\mu}$. The figure shows that for some earthquakes, the maximum displacement decreases as the mass ratio $\boldsymbol{\mu}$ increases, however there are other cases which do not show the same trend [11], and in same cases the displacement may increase with the increase of $\boldsymbol{\mu}$. 


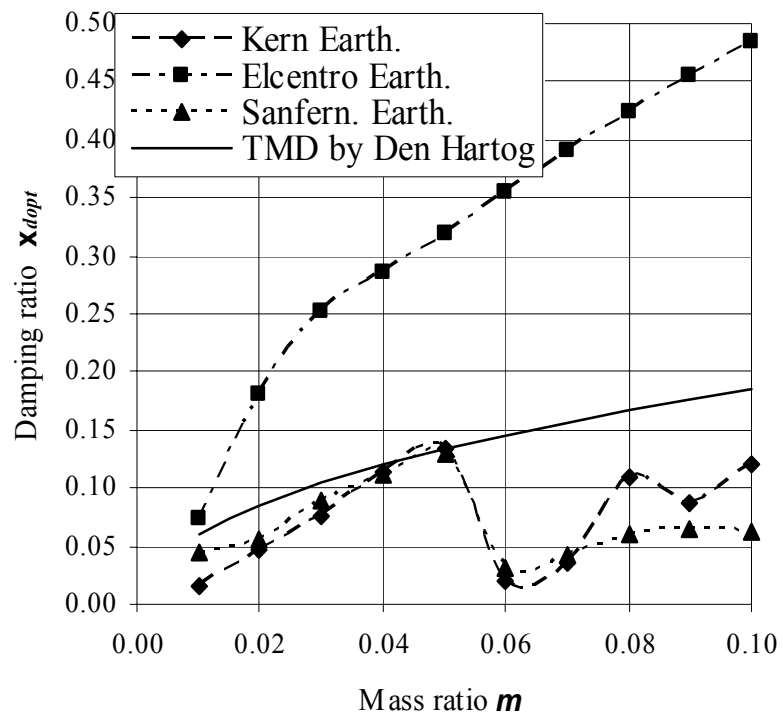

Figure 8: $\quad$ Variation of $\boldsymbol{\xi}_{\text {dopt }}$ with $\boldsymbol{\mu}$ for various earthquakes.

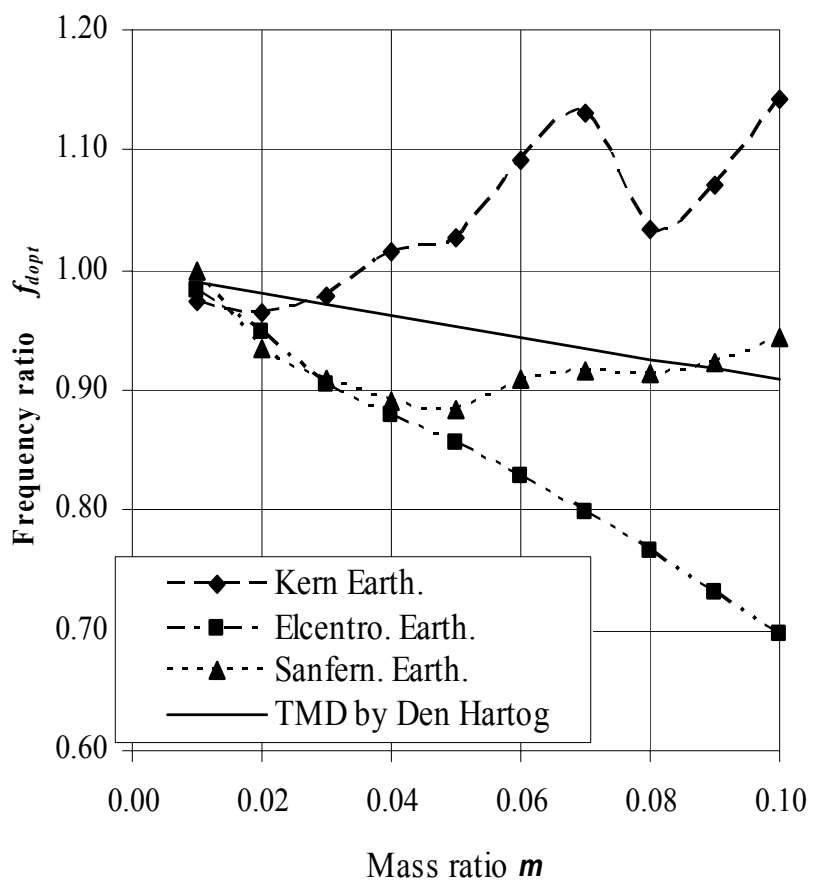

Figure 9: $\quad$ Variation of $\boldsymbol{f}_{\text {dopt }}$ with $\boldsymbol{\mu}$ for various earthquakes. 


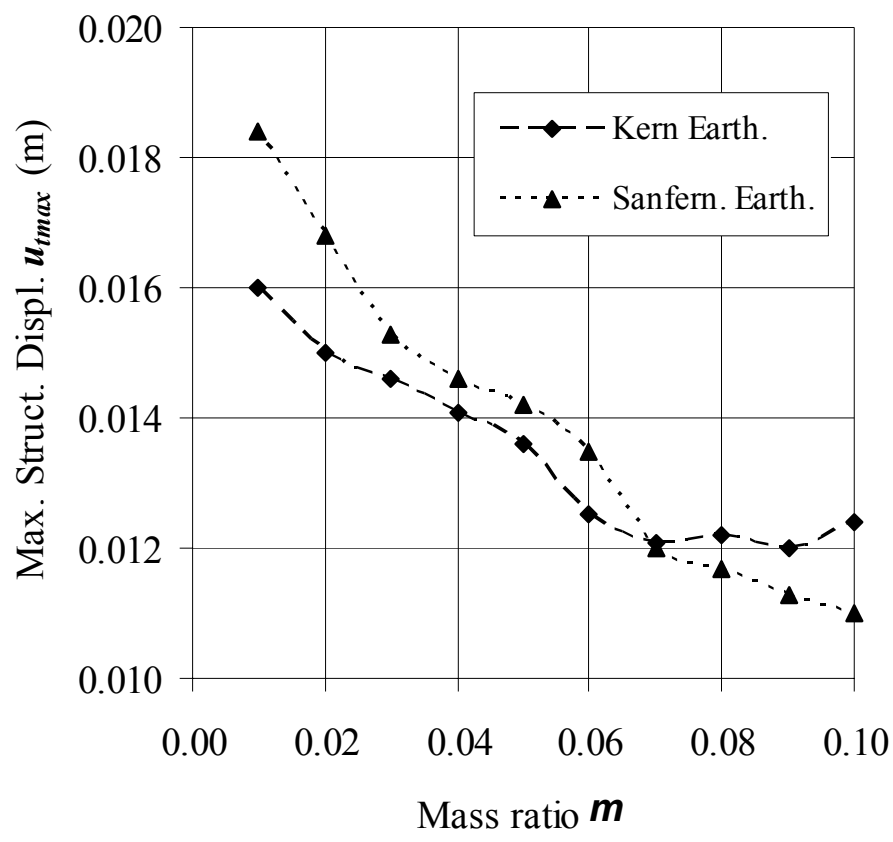

Figure 10: Figure 10: Variation of $\boldsymbol{u}_{\operatorname{tmax}}$ with $\boldsymbol{\mu}$ for various earthquakes.

The conclusion here is that the relation between maximum displacement $\boldsymbol{u}_{\text {tmax }}$ and mass ratio $\boldsymbol{\mu}$ is also affected by the earthquake type and there is no general trend for this relation as determined by Den Hartog or other similar methods.

\section{Conclusions}

The following main conclusions can be drawn from the present study:

- For a given SDOF structure, earthquake excitation and TMD mass, the present study method and software developed are capable to trace and compute the optimum values for $k_{d}$ and $c_{d}$.

- The TMD system designed according to the present study method is more effective in reducing maximum structure displacement than other methods, such as Deng Hartog. This is also found true for a wide range of earthquake excitations and structure frequencies.

- The efficiency of the TMD system designed according to the present study method and other methods are generally affected by the earthquake excitation. This means that the earthquake characteristics have an important effect on the TMD behavior and should be considered in the design process. 
- With the increase of research about predicting earthquake timeacceleration history, the importance of the present study will increase as an efficient method for designing TMD systems.

- For SDOF structures with TMD system designed according to the present study method, the relationship between $f_{\text {dopt }}$ (optimum tuning frequency) or $\boldsymbol{\xi}_{\text {dopt }}$ (optimum TMD damping ratio) with $\boldsymbol{\mu}$ (TMD mass ratio) is affected by the earthquake type and cannot be predicted by simple equation as in the Den Hartog or similar methods as shown.

- For some earthquakes, the maximum structure displacements decrease with the increase of $\boldsymbol{\mu}$ (as in the Den Hartog method). However, there are cases for other earthquakes that showed different trends. This implies that the earthquake type may affect the trend of the relationship between the maximum structure displacement and mass ratio $\boldsymbol{\mu}$.

\section{References}

[1] Den Hartog, J. P. Mechanical Vibration, McGraw-Hill, New York. N.Y., 1956.

[2] Warburton, G. B. and Ayorinde, E. O., Optimum absorber parameters for simple systems, Earthq. Engng Struct. Dynam, 8, pp 197-217, 1980.

[3] Tsai, H. C. and Lin, G. C., Optimum tuned mass dampers for minimizing steady-state response of support excited and damped systems, Earthq. Engng Struct. Dynam., 22, pp 957-973, 1993.

[4] Warburton, G. B., Optimal absorber parameters for various combinations of response and excitation parameters, Earthq. Engng Struct. Dynam, 10, pp 381-401, 1982.

[5] Rahul Rana and T. T. Soong, Parametric, study and simplified design of tuned mass dampers, Engineering Structures, 20(3), pp. 193-204, 1998.

[6] Sadek, F., Mohraz, B., Taylor, A. and Chung, R., A Method of Estimating The Parameters Of Tuned Mass Dampers For Seismic Applications, Earthq. Engng Struct. Dynam, 26, pp.617-635, 1997.

[7] Fengxin, Z., Yushan, Z. and Hongshan, L., Artificial ground motion compatible with specified ground shaking peaks and target response spectrum, Journal of Earthquake Engineering and Engineering Vibration, 1, pp 41-48, (2006).

[8] Abdalla, J. A. \& Y. M. Hag-Elhassam, Simulation of Earthquake Ground Motion for Generation of Artificial Accelerograms, ERES V, 2005.

[9] Varpasuo, P. H. A. \& Gelder, J. M. V., Generation of Design Ground Motion Time Histories for Lianyungang Nuclear Power Plant, ICOSSAR' 01, New port Beach, pp.17-22, 2001.

[10] MATLAB Version 7.10, The Language of Technical Computing, The Math Works, Inc. (2005).

[11] Al-Taweel, M. G., Optimum Design of Tuned Mass Damper Systems for Seismic Structures, MSc Thesis Submitted To The Faculty of Engineering, Al-Tahadi University, Sirte, Libya, 2007. 